\title{
Téoros
}

Revue de recherche en tourisme

\section{Un paysage à révéler, celui du Québec fantastique}

\section{Jules Lamarre et Louise Lamarre}

Volume 15, numéro 1, printemps 1996

URI : https://id.erudit.org/iderudit/1075055ar

DOI : https://doi.org/10.7202/1075055ar

Aller au sommaire du numéro

Éditeur(s)

Université du Québec à Montréal

ISSN

0712-8657 (imprimé)

1923-2705 (numérique)

Découvrir la revue

Citer cet article

Lamarre, J. \& Lamarre, L. (1996). Un paysage à révéler, celui du Québec fantastique. Téoros, 15(1), 44-45. https://doi.org/10.7202/1075055ar d'utilisation que vous pouvez consulter en ligne.

https://apropos.erudit.org/fr/usagers/politique-dutilisation/ 


\title{
UN PAYSAGE À RÉVÉLER, CELUI DU QUÉBEC FANTASTIQUE
}

\author{
Jules Lamarre et Louise Lamarre
}

Jules Lamarre, Ph.D.,
géographe
Chargé de cours à l'UQAR
Module de géographie
et
Louise Lamarre, professeure
Département de cinéma
Université Concordia

physiques, riveres, vhes, leur accole aussitôt une certaine charge mentale (L.-E. Hamelin, 1975). Le nom de lieu force la connection, pour ainsi dire, entre un lleu et ce que l'on salt à son sujet. Aussitôt, des noms comme New York, Montréal, Rimouski font référence à des lleux auxquels on n'a comme pas le choix d'associer une atmosphère, une réputation, des connalssances de toutes sortes, vrales et fausses. Même si on n"a Jamals mis les pleds à Chibougamau, nl vu de ses propres yeux les portes de l"enfer sur la rivière Rimouski, prononcer ces seuls mots met l'Imagination en marche qui confère à ces lleux des attributs, Invente des histoires à leur sujet.

De ce polnt de vue, les toponymes, et plus précisément leur caractère évocateur, s"avèrent une ressource intéressante à explolter d'un point de vue touristique. Au moyen du cinéma, ce médium qui rend réels les frults de l'imaginaire, il est possible de mettre en Images, d" wimaginer* à partir des noms de lleux eux-mêmes, des paysages à falre connaître aux touristes d'lcl et d'allleurs. En tenant compte du caractère évocateur des noms de lleux, Jean Polrier $(1982$, p. 7) a Identiflé trois types principaux de formations toponymiques dans la nomenclature géographlque québécolse. Il y a des noms descriptufs (lac rond, lac carré), des noms de femmes ou d'hommes (Drummondville), ainsi que des noms qul ont à voir avec nos croyances rellgleuses, comme tous ces noms de Saintes et de Saints que l'on a donné à une foule de municlpalltés. Puls vlennent quelques catégories moins souvent représentées, mais combien plus intéressantes pour notre propos, comme celle des légendes.

Les enquêtes toponymlques révèlent que plusieurs toponymes au Québec tirent leur origine de légendes ou blen qu'ils ont eux-mềmes inspiré de telles légendes après avoir été mis en usage. Dans les deux circonstances, les légendes toponymiques rapportent des événements extraordinaires qui se seralent déroulés dans des endrolts précls. Ces récits tirent profit diune géographie locale dont les éléments sont magiques. Ce sont souvent de petits chef-d'ocuvres fantastlques racontant des mésaventures vécues par des humains par trop téméralres ayant visité des lieux hantés au mauvais moment... On y retrouve des fées, des diables et des lutins en abondance.
SI les noms de lleux possèdent la propriété de créer des paysages (TIlley, 1994, p. 19), les noms de lleux qul rappellent des légendes toponymlques, par exemple, suggènent l'existence Icl d'un paysage blen particulier, celle d'un paysage fantastique, dont la connalssance est à notre portée: Il ne demande qu'à être *révélé *. Pour le mettre en évidence, Il y aurait lieu de recenser d'abord les toponymes québécols évoquant le fantastlque, de les classer par thèmes et d'analyser ensulte les densités de répartituon des éléments appartenant ả chacune de ces catégorles dans le but de repérer les aires d'extension spatlale de ces thèmes. Ce seralt une façon simple d'obtenlr une carte des réglons fantastiques d'icl, une carte du Québec fantastique. Dans un second temps, le cinéma pourrait prendre la relève en mettant en image une légende toponymlque représentative du faclès fantastıque de chacune des réglons. On pourrait alnsı créer de nouveaux attraits touristiques qui non seulement pourralent faire connaitre un aspect particullèrement fasclnant de la culture québécolse à l'étranger mals aussl intéresser les Québécols et Québécolses à visiter leur propre paysage fantastique.

Peu de gens d'lcl connalssent l'existence des légendes toponymlques, mis à part les toponymistes. Pourtant, ces légendes appartlennent à notre patrimolne. Lorsque l'on se falt raconter une de ces légendes qui se rattachent à un endroit que l'on visite, II n'est plus possible par la sulte de voir cet endroit de la même façon. Le lleu le plus banal, du molns en apparence, prend alors les dimensions dun monument. L'imaginaire prend le dessus. Grâce au clnéma II devlent possible de transformer des lleux en monuments et de les exporter en incitant les gens d'allleurs à venir les volr.

Ici, au Québec, les films pourralent être présentés dans les divers musées régionaux et sur des traverslers. Un Itinéraire touristique ponctué de visionnement de films toponymiques pourralt alors donner accès au visage d'un Québec fantastlque insoupçonne. Nous avons mis ce projet en route.

Voici d'ailleurs un bel exemple de légende toponym lque:

\section{L'Arbre-Sec-à-Carnolet}

La paroisse de Saint-Laurent de l'Ile d'Orléans, fondée en 1679. s"appelalt à l'origine Saint-Paul-de-l'Arbre-Sec. Ge toponyme rappelle l'étrange aventure qul seralt arrlvée dans les parages au vlolonneux Françols Gosselin et à son volsin, le menuisier Carnolet.

On raconte qu'en revenant d'une de ces longues velllées où II falsalt danser les villageols, Gosselin, le violonneux bossu, s'était endormi le long de la route près d'un rocher. Révellé́ 
à minult par des bruits étranges, le bossu voulut s'enfuir quand une bande de lutins lul barra la route. Ceux-cl lul proposèrent alors de les falre danser toute la nult au son de son vlolon en échange de quol, s'il s'acquittalt blen de sa tâche, Il seralt blen récompensé. Et Gosselln de s'exécuter, parce qu'll n'avalt pas le cholx, et de falre danser les lutins jusqu'au matin. Au lever du jour le chef des lutins s"avança alors pour luire faire une offe en ces termes *Fortune ou beauté?*. Françols réfléchit un moment et, portant la main à sa bosse, répondlt: -Voyez, si vous pouviez m'enlever ce paquet, ma fol! J'en serals très heureux. Françols eut à peine le temps de remercler les lutins qu"lls avalent tous disparus. Plus tard en arrivant chez lul, Gosselin rencontra son volsin Carnolet qul, ne voyant plus la bosse de Gosselin, crut aussitốt que le violonneux avalt vendu son âme au dlable. Colncé, le vlolonncux dut alors lul raconter son aventure de la nult précédente.

Le solr même, à son tour Carnolet déclde de se rendre à l'endrolt que lul avalt Indlqué Gosselin en emportant avec lul cette flûte dont Il joualt à l'occasion. Sur le coup de minult, les lutins réapparurent et obllgèrent également Carnolet ả les falre danser toute la nut au son de son instrument, ce qui, blen sùr, n'était pas pour lui déplalre. Au matin, le chef des lutins lui proposa également de cholsir entre la fortune et la beauté. Sans réfléchir. Carnolet répondit aussitôt: -Donnez-mol donc, messleurs, ce dont le violonneux n'a pas voulu*. Et pendant que les lutins disparalssalent, la bosse de
Gosselin se mit à pousser sur le dos de Carnolet! Tremblant de colère, Carnolet revint la nult sulvante pour exiger des explications. Gredin! que m'as-tu planté là sur le dos?. lanç-L-II au chef des lutins. *Ce que tol-même m'a demandé*, de lul répondre le lutin. Fou de rage. le menulsier s'élanca alors vers les lutins en brandissant sa flûte pour les frapper. Mais son bras reste en l'alr, flgé, sa volx s'étrangle dans sa gorge; et II sent ses pleds prendre racine, sa peau durclr et se fendiller comme l'écorce d'un viell arbre, ses bras s'allonger en forme de branches.

On ne revit jamals Carnolet. Passant au mème endrolt quelques Jours plus tard, le violonneux remarqua qu'Il s'y trouvalt un vleil arbre tout tordu et sec qul n'y étalt pas auparavant. Au bout d'une branche pendant encore la flute de Carnolet. Par la suite, l'endrolt fut désigné par le nom d'Arbre-Sec-à-Carnolet (lle d'Orléans)

\section{Références}

HAMELIN, Louls-Edmond, Nordiché canadlenne, Montréal, Hurtubise-HMH, 1975, 458 p.

POIRIER, Jean, Regards sur les noms de Ileux, Commisslon de toponymie du Québec. Études et recherches toponymiques, no 3, 1982, $174 \mathrm{p}$.

TILLEY, Christopher. A Phenomenology of Landscape, Provldence (Ë-U.), Berg Publishers, 1994, 221 p.

\section{Votre inscription au BOTTIN TOURISTIQUE 1996}

\section{L'industrie du tourisme culturel, récréatif et d'affaires au Québec}

Ce Bottin, qui paraît à la fin de mai, fournit les coordonnées postales et téléphoniques, secteurs d'activité et divers renseignements sur les intervenants touristiques au Québec. Tiré à 2500 exemplaires, ce Who's who est distribué gracieusement et largement dans les milieux touristiques, associatifs, économiques et gouvernementaux du Québec, du Canada et de l'étranger.

En s'y inscrivant, on s'assure d'une plus grande visibilité et on met en valeur les services, produits et attraits de son entreprise ou organisme tout en le personnalisant. Les frais d'inscription sont de $\mathbf{5 0} \mathbf{5}$. En retour, nous vous inscrivons dans le Bottin, nous vous en expédions un exemplaire gratuit et nous vous abonnons au périodique InfoCongrès.

Pour s'inscrire ou pour obtenir de plus amples renseignements, s'adresser $\dot{a}$

QUÉBEC DANS LE MONDE / C.P. 8503 / Sainte-Foy (Québec) / G1V 4N5

Tél. (418) 659-5540 ou Fax: (418) 659-4143 